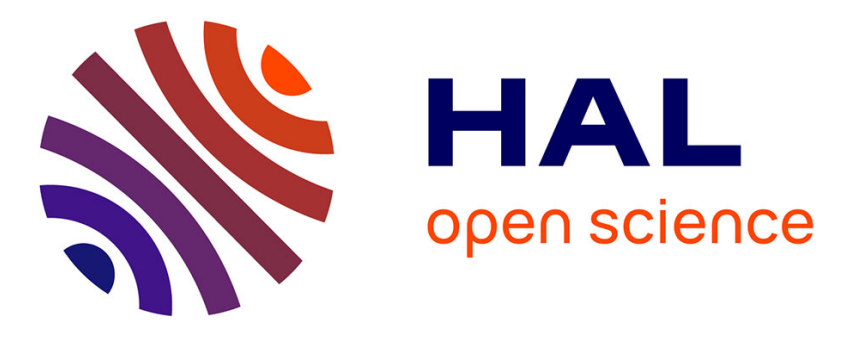

\title{
Représentations Systèmes Multi-Machines (SMM) de machines polyphasées
}

Eric Semail, Farid Meibody-Tabar, Mohamed Fouad Benkhoris, Hubert Razik,

Maria Pietrzak-David, Eric Monmasson, Alain Bouscayrol, Bernard Davat, Philippe Delarue, Bernard de Fornel, et al.

\section{To cite this version:}

Eric Semail, Farid Meibody-Tabar, Mohamed Fouad Benkhoris, Hubert Razik, Maria Pietrzak-David, et al.. Représentations Systèmes Multi-Machines (SMM) de machines polyphasées. Représentations Systèmes Multi-Machines (SMM) de machines polyphasées, 2005, 8 (2), pp.221-239. 10.3166/rige.8.221-239 . hal-01107789

\section{HAL Id: hal-01107789 \\ https://hal.science/hal-01107789}

Submitted on 3 Feb 2015

HAL is a multi-disciplinary open access archive for the deposit and dissemination of scientific research documents, whether they are published or not. The documents may come from teaching and research institutions in France or abroad, or from public or private research centers.
L'archive ouverte pluridisciplinaire HAL, est destinée au dépôt et à la diffusion de documents scientifiques de niveau recherche, publiés ou non, émanant des établissements d'enseignement et de recherche français ou étrangers, des laboratoires publics ou privés. 


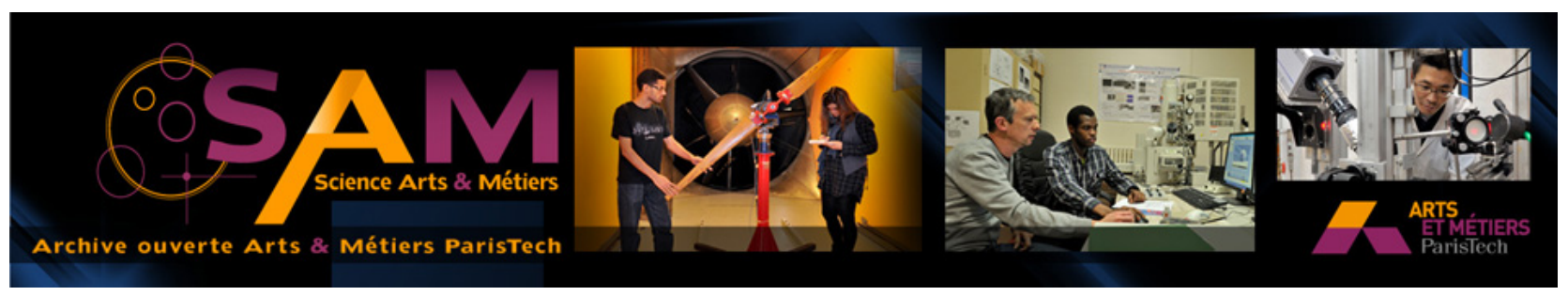

\section{Science Arts \& Métiers (SAM)}

is an open access repository that collects the work of Arts et Métiers ParisTech researchers and makes it freely available over the web where possible.

This is an author-deposited version published in: http://sam.ensam.eu

Handle ID: .http://hdl.handle.net/10985/9260

\section{To cite this version :}

Eric SEMAIL, Farid MEIBODY-TABAR, Mohamed BENKORIS, Hubert RAZIK, Maria PIETRZAKDAVID, Eric MONMASSON, Alain BOUSCAYROL, Bernard DAVAT, Philippe DELARUE, JeanPaul HAUTIER, Jean-Paul LOUIS, Serge PIERFEDERICI - Représentations Systèmes MultiMachines (SMM) de machines polyphasées - Représentations Systèmes Multi-Machines (SMM) de machines polyphasées - Vol. 8, n², p.221-239-2005 


\title{
Représentations Systèmes Multi-Machines (SMM) de machines polyphasées
}

\author{
E. Semail ${ }^{1}$, F. Meibody-Tabar ${ }^{2}$, M. F. Benkhoris ${ }^{3}$, H. Razik ${ }^{2}$, M. \\ Pietrzak-David $^{4}$, E. Monmasson ${ }^{5}$, A. Bouscayrol ${ }^{1}$, B. Davat ${ }^{2}$, Ph. $^{2}$ \\ Delarue $^{1}$, B. de Fornel ${ }^{4}$, J. P. Hautier ${ }^{1}$, J. P. Louis ${ }^{5}$, S. Pierfederici ${ }^{2}$ \\ L2EP, Laboratoire d'Electrotechnique et d'Electronique de Puissance de Lille \\ http://www.univ-lille1.fr/l2ep/_ Alain.Bouscayrol@univ-lille1.fr \\ ${ }^{1}$ L2EP Lille, UPRES EA 2697, Université de Lille 1 (USTL), 59655 Villeneuve \\ d'Ascq cedex, Eric.Semail@lille.ensam.fr \\ ${ }^{2}$ GREEN, UPRES A 7037, ENSEM, 2 avenue de la Forêt de Haye, 54600 \\ Vandoeuvre les Nancy, Farid.Meibody-Tabar@ensem.u-nancy.fr, \\ ${ }^{3}$ IREENA, Boulevard de l'Université, BP 406, 44602 Saint-Nazaire, France, \\ mohamedfouad.benkhoris@polytech.univ-nantes.fr \\ 4 LEEI, UMR 5828, ENSEEIHT, 2 rue Camichel, 31071 Toulouse cedex, \\ Maria.David@leei.enseeiht.fr, \\ 5 SATIE, UMR 8029, IUP GEII de Cergy, rue d'Eragny, Neuville sur Oise, 95031 \\ Cergy-Pontoise Cedex, Eric.Monmasson@iupge.u-cergy.fr, \\ 1,2,3,4,5Projet SMM du GdR ME MS, Alain.Boucayrol@univ-lille1.fr, URL: \\ http://www.univ-lille1.fr/l2ep/web-smm.htm
}

\footnotetext{
RÉSUMÉ. Cet article présente le principe de décomposition de machines polyphasées en machines fictives monophasée et diphasées non couplées magnétiquement. Après la description de la méthodologie de décomposition SMM (Systèmes Multimachines Multiconvertisseurs), deux cas sont étudiés. Une machine synchrone pentaphasée, est d'abord analysée avec son modèle de machines équivalentes. Un second cas plus original est ensuite étudié : deux machines pentaphasées connectées en série et alimentées par un onduleur 5 bras.

ABSTRACT. This paper presents the equivalence of multi-phase machines with a set a of 1-phase and 2-phase machines with no magnetic couplings. Two cases are then studied. First, a 5 phase machine supplied by a Voltage Source Inverter(VSI) is analyzed. Then, a model is established for a single 5-leg VSI supplying two 5-phase machines whose windings are connected in series.

MOTS-CLÉS: multimachine, multiconvertisseur, polyphasée,pentaphasée.

KEY WORDS: Multi-machine multiconverter, multi-phase, multimotor drives, series connection.
} 


\section{Introduction}

Depuis un certain nombre d'années, les machines polyphasées trouvent un regain d'intérêt [TOL-00] [LyR-01] [COA-01]. En effet, les progrès de l'électronique de puissance permettent maintenant d'assurer des alimentations adaptées [SEM-00]. De plus, un grand nombre de phases permet une segmentation de puissance qui répartit les contraintes sur plusieurs composants [Mou-99]. Cela permet, entre autre, d'utiliser des composants de puissance fonctionnant avec une fréquence de commutation élevée, et ainsi, de réduire les taux harmoniques des courants et l'ondulation du couple [Mou-99]. Enfin, ces machines autorisent des modes de marches dégradées lors d'une mise en défaut d'un bobinage ou d'un composant de puissance [JAH-80]. Cependant, ces divers avantages ne doivent pas occulter la complexité de leur commande, tant en mode normal qu'en mode dégradé [TOL-00].

Le projet SMM (Systèmes Multimachines Multiconvertisseurs) du GdR SDSE puis du GdR ME2MS ${ }^{1}$, a travaillé sur l'étude, la représentation synthétique et la commande de systèmes de conversion électromécanique composés de plusieurs machines et / ou convertisseurs statiques [SMM-00A]. Dans la classification des SMM, les machines polyphasées correspondent à des systèmes à couplage magnétique. Elles sont ainsi perçues comme des machines "classiques" mettant en commun une carcasse magnétique afin de produire un couple commun à partir de plusieurs sources d'alimentation. Une étude préalable sur la généralisation du vecteur d'espace avait démontré qu'un système polyphasé pouvait se décomposer en plusieurs systèmes diphasés orthonormés pouvant être gérés de manières indépendantes [Sem-00]. Les travaux récents du projet SMM ont combiné les deux approches pour décomposer une machine polyphasée en machines fictives équivalentes possédant des propriétés intéressantes pour la commande [Sem-01]. Diverses études de machines pentaphasées ont été réalisées avec cette approche [Kes-02], [Mar-02], [Rob-02].

Cette communication présente le principe de décomposition de machines polyphasées en machines fictives monophasée et diphasées non couplées magnétiquement. Après la description de la méthodologie de décomposition, deux cas sont étudiés. Une machine synchrone pentaphasée, cas considéré maintenant comme classique, est d'abord analysée avec son modèle de machines équivalentes. Un second cas plus original est ensuite étudié : deux machines pentaphasées connectées en série et alimentées par un onduleur 5 bras [Gat-00].

\footnotetext{
${ }^{1}$ Groupes de Recherche du CNRS "Sûreté et Disponibilité des Systèmes Electriques" (19982001) et "Maîtrise de l'Energie Electrique du Matériau au Système" (2002-2005)
} 


\section{Décomposition en Machines Fictives Equivalentes}

\subsection{Formalisme SMM [SMM-00A]}

Le formalisme SMM correspond à une représentation synthétique des conversions électromécaniques par des éléments interconnectés selon le principe de l'action et de la réaction. On distingue alors les éléments suivants (Figure 1) :

- sources (générateur ou récepteur d'énergie, ovales)

- convertisseurs électriques (carrés),

- machines électriques (cercles),

- convertisseurs mécaniques (triangles).

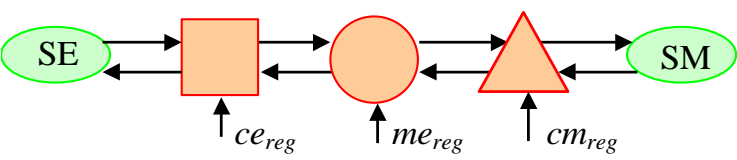

Figure 1. Chaîne élémentaire de conversion électromécanique

Des éléments de couplages sont introduits pour prendre en compte la distribution de l'énergie sur différentes chaînes de conversion (Figure 2). Représentés par des pictogrammes imbriqués, ils sont considérés comme des entités "classiques" partageant une ressource physique commune et induisant une variable commune.

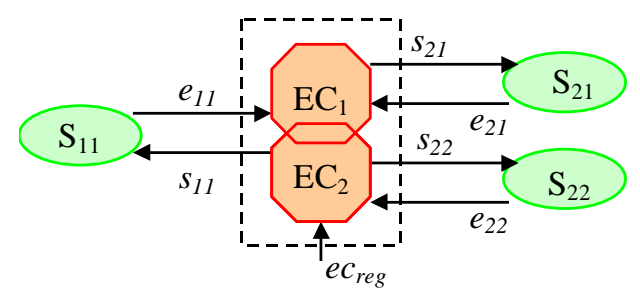

Figure 2. Exemple d'élément de couplage

\subsection{Généralisation du vecteur d'espace [Sem-00][Kes-03]}

Une machine polyphasée est composée de $n$ bobinages déphasés spatialement de $2 \pi / n$ et alimentés par des tensions déphasées temporellement de $2 \pi / n$. Ces machines sont caractérisées par un couplage magnétique entre phases.

Pour les machines triphasées, la transformation de Park ou la notion de vecteur d'espace (dimension 2) permet de trouver des bobinages fictifs équivalents qui simplifient l'étude. En effet, le couplage magnétique est réduit par des axes de travail orthogonaux. Cette opération correspond en fait à un changement de base. 
La généralisation de la méthode du vecteur d'espace permet de définir un changement de base de dimension $n$, impliquant une simplification de l'étude de la machine par la diagonalisation de la matrice inductance. On montre alors que ce changement de base conduit à des sous-espaces vectoriels orthogonaux de dimension 2 ou 1. Chaque sous-espace peut ainsi être indépendant. La notion de machine fictive de dimension 1 ou 2 est alors introduite.

\subsection{Application aux machines polyphasées [SEM-03]}

La disparition du couplage magnétique induit par la transformation du vecteur d'espace généralisé, fait apparaître un couplage électrique et un couplage mécanique (Figure 3).

Le couplage électrique correspond à la transformation mathématique qui lie les phases d'origine aux phases fictives, $v_{k}$ étant la tension aux bornes de la phase $\mathrm{n}^{\circ} k$ de la machine. Quant au couplage mécanique, il est en fait relativement simple car il définit le couple total comme étant la somme des couples des machines fictives.

Ainsi, on transforme donc un couplage magnétique contraignant et difficile à gérer en couplages électriques et mécaniques simples. De plus, les machines fictives équivalentes sont de dimension 1 ou 2 . Or ce type de machine fictive est bien connu : bobinages équivalents dans le repère $(\mathrm{d}, \mathrm{q})$ de Park par exemple.
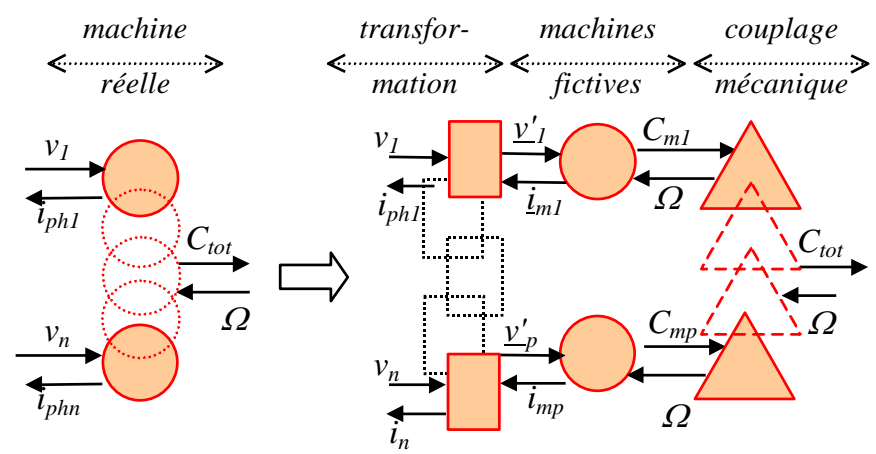

Figure 3. Couplages de la machine réelle et des machines fictives

\section{Application à un Cas Standard}

\subsection{Structure étudiée}

Une machine synchrone pentaphasée est alimentée par 5 onduleurs monophasés (Figure 4). Chaque phase est ainsi alimentée par son propre onduleur ce qui permet 
un fonctionnement en mode dégradé lors d'un défaut d'un composant de puissance ou d'un bobinage.

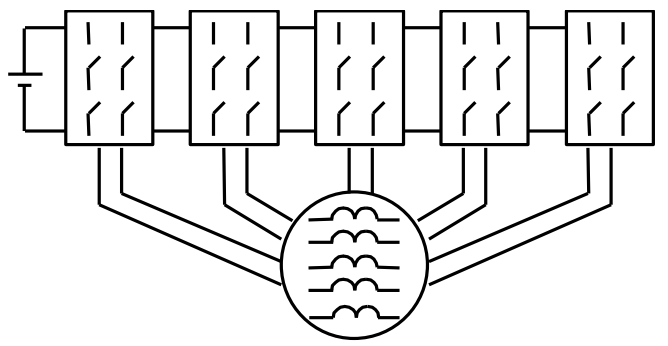

Figure 4. Onduleurs monophasés pour machine synchrone pentaphasée

Une alimentation de type MLI intersective classique fait apparaître de fortes ondulations de courants (Figure 5), autour de la référence sinusoïdale demandée. Ces ondulations sont inattendues à la vue du rapport faible entre la période de MLI et la constante de temps définie classiquement par $L_{c} / R$ (avec $R$ résistance d'une phase et $L_{c}$ inductance cyclique dans le cadre d'une approche au premier harmonique). Par l'approche originale proposée, il apparaît que d'autres constantes de temps beaucoup plus faibles doivent être prises en compte afin de prédéterminer quantitativement l'amplitude de ces ondulations.

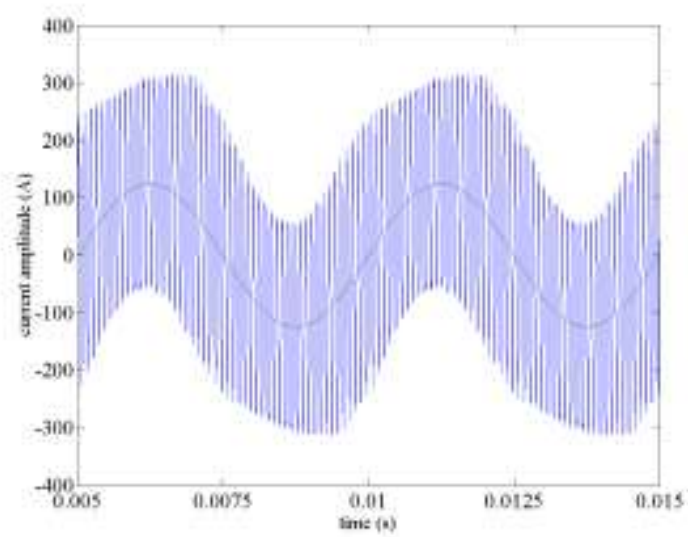

Figure 5. Courant dans une phase de la machine

\subsection{Représentation SMM}

La représentation SMM du système est composée des 5 onduleurs monophasés (carrés) et de la machine pentaphasée considérée comme 5 machines partageant la même carcasse (cercles imbriqués). Par souci de simplification, la source de tension 
continue est supposée idéale. Elle est alors représentée par 5 sources électriques qui fournissent la même tension $V_{D C}$ (Figure 6) (règle de simplification des couplages [SMM-00B]).

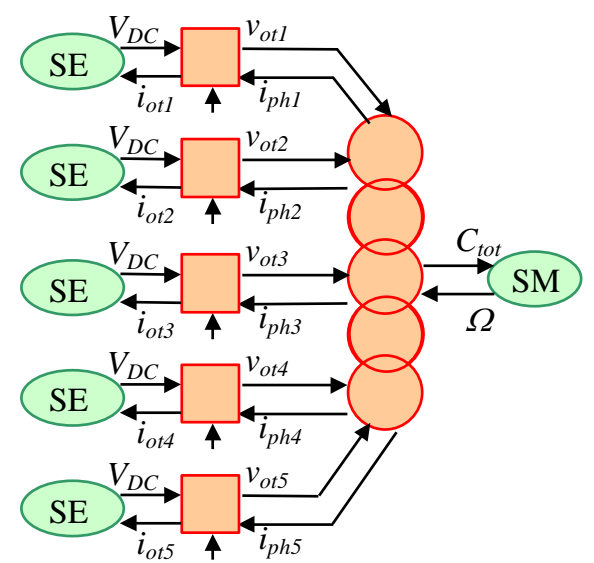

Figure 6. Description SMM du système étudié avec machine réelle

Les hypothèses suivantes sont adoptées pour modéliser la machine:

- toutes les phases sont identiques et régulièrement décalées par un angle $\alpha=2 \pi / 5$;

- pas d'effet de réluctance variable (pôles lisses);

- pas d'effet de saturation (circuit magnétique linéaire).

Toutes les quantités concernant la phase $k$ sont écrites $x_{k}$. La machine pentaphasée est représentée schématiquement sur la Figure 7.

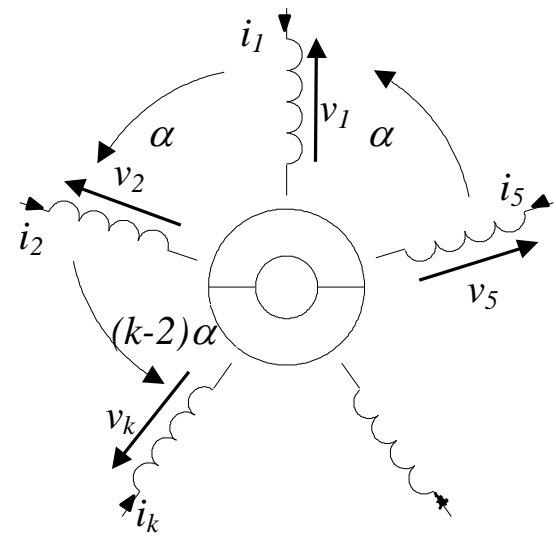

Figure 7. Présentation de machine synchrone de pentaphasée

Considérons la matrice [L] des inductances propres : 


$$
[L]=\left[\begin{array}{ccccc}
L_{0} & M_{1} & M_{2} & M_{2} & M_{1} \\
M_{1} & L_{0} & M_{1} & M_{2} & M_{2} \\
M_{2} & M_{1} & L_{0} & M_{1} & M_{2} \\
M_{2} & M_{2} & M_{1} & L_{0} & M_{1} \\
M_{1} & M_{2} & M_{2} & M_{1} & L_{0}
\end{array}\right]
$$

Son caractère symétrique et circulant permet de mettre en évidence 3 valeurs propres $L_{c k}$, appelées inductances cycliques, et 3 espaces propres orthogonaux entre eux : deux plans et une droite.

On décompose de façon unique tout vecteur en une somme de 3 vecteurs appartenant chacun à l'un des 3 sous-espaces. A chaque plan on associe, un phaseur complexe, vecteur de dimension 2. A la droite, on associe un scalaire, vecteur de dimension 1. On obtient finalement les 3 équations suivantes faisant apparaître chacune une valeur propre $L_{c 0}, L_{c 1}, L_{c 2}$ :

$$
\begin{aligned}
& v_{0}=R i_{0}+L_{c 0} \frac{d i_{0}}{d t}+e_{0} \\
& \underline{v}_{d q 1}=R \underline{i}_{d q 1}+L_{c 1} \frac{d}{d t} \underline{i}_{d q 1}+\underline{e}_{d q 1} \\
& \underline{v}_{d q 2}=R \underline{i}_{d q 2}+L_{c 2} \frac{d}{d t} i_{d q 2}+\underline{e}_{d q 2}
\end{aligned}
$$

Étant donné que les 3 sous-espaces propres sont orthogonaux, le bilan de puissance peut se décomposer en une somme de trois bilans élémentaires indépendants. Il est alors permis d'associer à chaque sous-espace propre une machine fictive participant à la création du couple de la machine réelle. En considérant que toutes les machines fictives tournent à la même vitesse $\Omega$, il vient que le couple total est simplement la somme des trois couples des machines fictives:

$$
C_{t o t}=C_{m p}+C_{m s}+C_{m h}
$$

Le couplage mécanique est donc très simple et on obtient alors la représentation proposée sur la Figure 8 où le changement de base est représenté par un couplage électrique. 


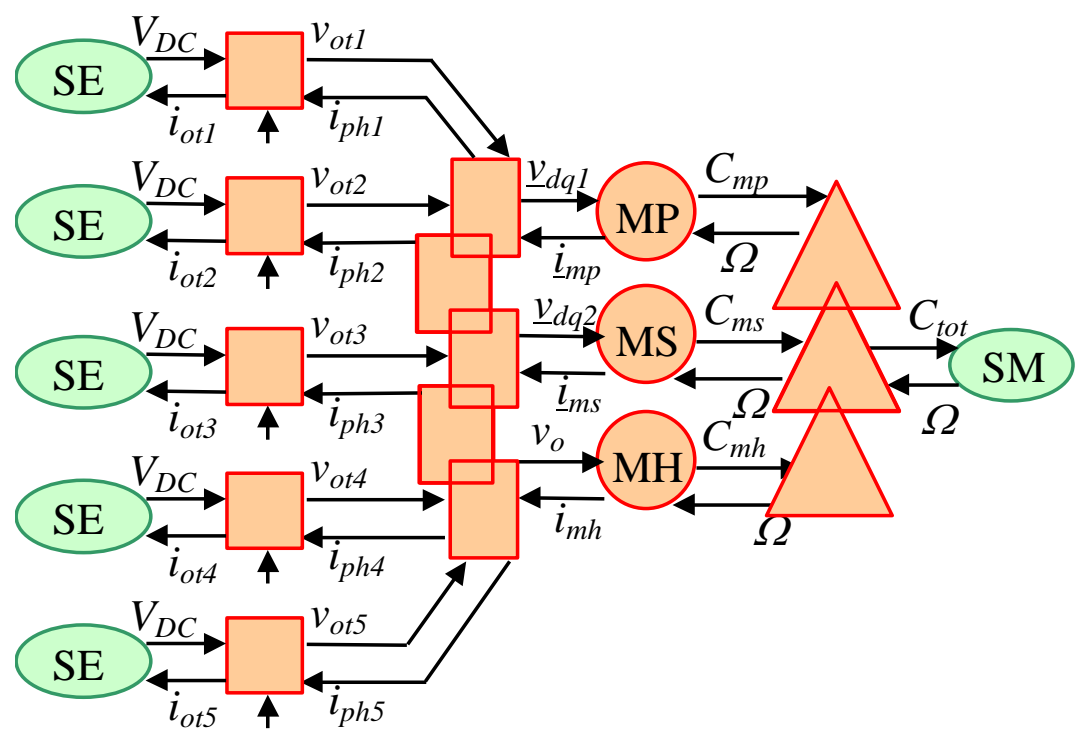

Figure 8. Description SMM du système étudié avec machines fictives

L'analyse de ces trois machines met en évidence, dans le cas d'une force électromotrice sinusoïdale, qu'une seule des 3 machines peut produire du couple. On appelle machine principale cette machine diphasée qui est liée à l'harmonique de rang 1 et dont l'inductance cyclique est notée $L_{c l}$.

Si par contre les forces électromotrices ne sont pas sinusoïdales alors chaque machine peut contribuer au couple, en fonction de l'amplitude de sa force électromotrice [Kes-02]. Ainsi, la deuxième machine diphasée pourra produire du couple principalement grâce aux harmoniques de rang 3 et 7 . Quant à la machine monophasée, la production de son couple pulsatoire est liée aux multiples de rang 5 .

On a donc ainsi remplacé le couplage magnétique par un couplage électrique simple (matrice de transformation) et un couplage mécanique simple (association des couples). Cette représentation va être utilisée dans un premier temps pour l'analyse des résultats obtenus dans le cas de l'alimentation MLI intersective. On projette pour cela le vecteur courant dans les 3 sous espaces propres définis pour obtenir les courants des machines fictives (Figure 9, Figure 10, Figure 11). 


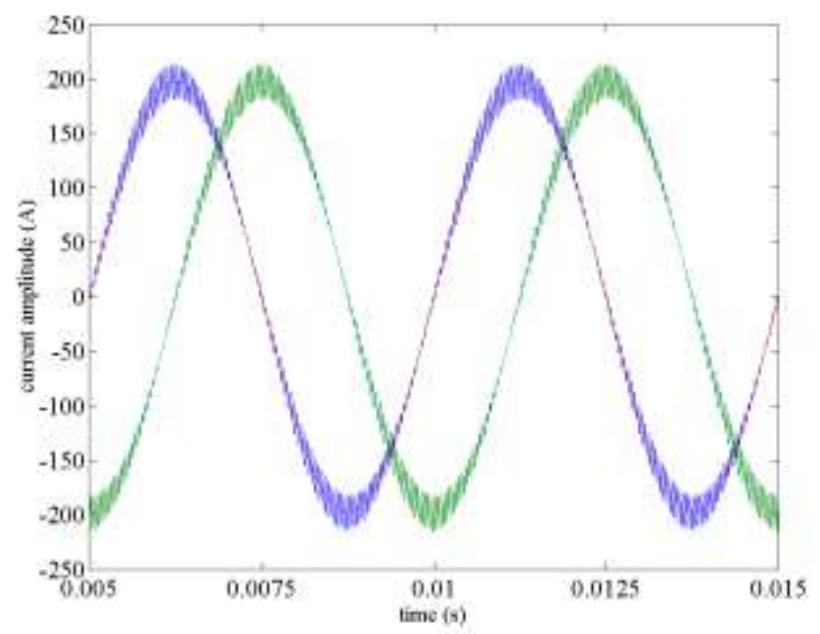

Figure 9. Courants dans la machine principale, MLI intersective

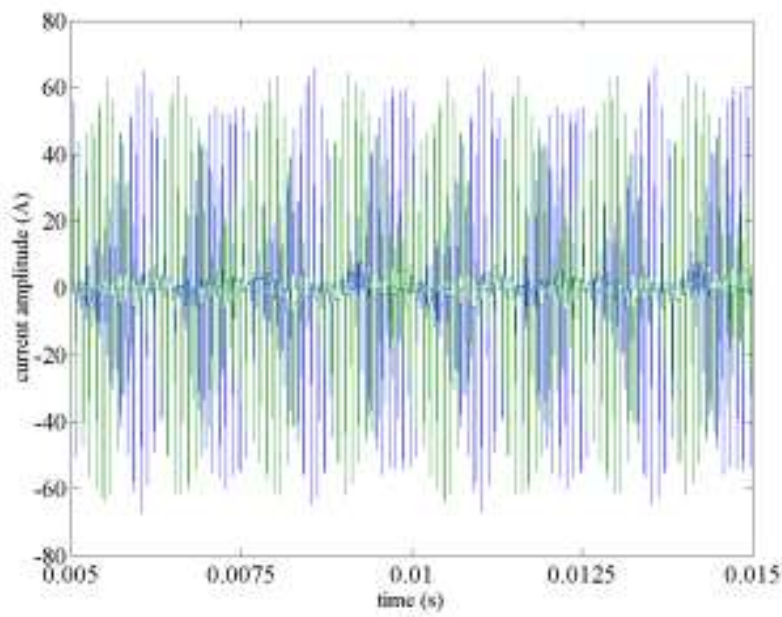

Figure 10. Courants dans la machine secondaire, MLI intersective 


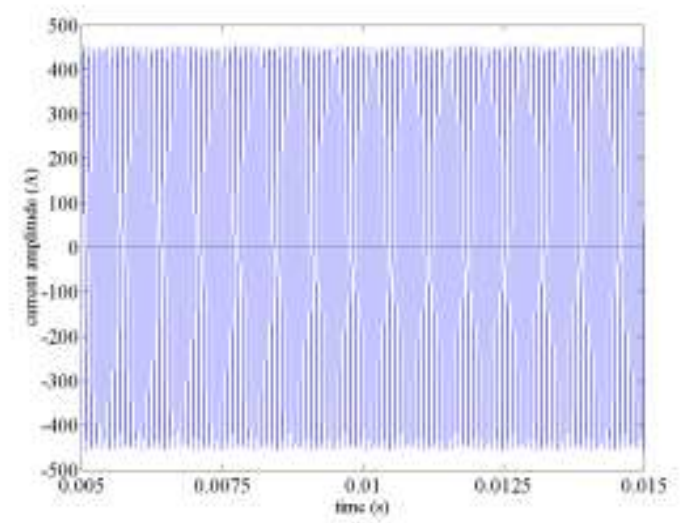

Figure 11. Courant dans la machine homopolaire, MLI intersective

Il apparaît alors que le taux d'ondulation des courants dans la machine principale est faible, conformément au rapport entre période de MLI et constante de temps $\mathrm{L}_{\mathrm{c} 1} / \mathrm{R}$. Par contre, il est manifeste que les constantes de temps des machines secondaire et homopolaire $\mathrm{L}_{\mathrm{c} 2} / \mathrm{R}$ et $\mathrm{L}_{0} / \mathrm{R}$ sont plus faibles que celle de la machine principale. Même si ces dernières ne sont pas alimentées aux valeurs moyennes, elles le sont aux valeurs instantanées, des courants parasites s'y développant alors.

\subsection{Commande déduite}

La commande de la machine permet de contrôler le couple qu'elle fournit. C'est donc un couple $C_{\text {tot-ref }}$ qui sera considéré. La présence du couplage mécanique met immédiatement en évidence alors la nécessité d'élaborer trois consignes de couple, une par machine fictive, à partir d'une seule consigne. La relation étant non bijective, il est nécessaire de définir des critères de répartition entre les différentes machines [SMM-03].

L'analyse des potentialités des machines secondaire et homopolaire met en évidence, dans la mesure où leurs forces électromotrices sont de faible amplitude par rapport à celle associée à la machine principale, que ces machines ne peuvent produire que peu de couple [Kes-02]. En revanche, leurs faibles impédances vis à vis de l'alimentation en tension est à l'origine de l'apparition de courants de forte amplitude, courants parasites dans la mesure où ils ne produisent pas de couple.

De cette représentation et de cette analyse on peut proposer une commande des trois machines fictives [MAR-02]. Une clé de répartition des couples de référence peut être la suivante :

- un couple de référence nul pour la machine secondaire et la machine homopolaire ;

- un couple de référence égal au couple total $C_{\text {tot-ref }}$ pour la machine principale ; 
Par ailleurs, l'alimentation des machines secondaire et homopolaire est choisie de façon à réduire l'amplitude des courants parasites qui s'y développent: les vecteurs caractéristiques de l'onduleur que l'on choisit d'utiliser pour cette commande sont ceux dont la composante homopolaire est nulle. A tout instant, la machine homopolaire n'est ainsi plus alimentée : il n'y a ainsi pas de courants qui s'y développent. Il faut donc fournir une représentation des 5 onduleurs monophasés pour chaque machine fictive. Il suffit pour cela de projeter les vecteurs tensions liés à l'état des interrupteurs dans les différents espaces propres des machines fictives.

Pour simplifier la mise en évidence de la commande, on prendra dans la suite le cas d'une machine à forces électromotrices sinusoïdales. Les forces électromotrices des machines secondaire et homopolaire sont alors nulles. Les équations en tension deviennent alors :

$$
\begin{aligned}
& v_{0}=R i_{0}+L_{c 0} \frac{d}{d t} i_{0} \\
& \underline{v}_{d q 1}=R \underline{i}_{d q 1}+L_{c 1} \frac{d}{d t} \underline{i}_{d q 1}+\underline{e}_{d q 1} \\
& \underline{v}_{d q 2}=R \underline{i}_{d q 2}+L_{c 2} \frac{d}{d t} \underline{i}_{d q 2}
\end{aligned}
$$

L'observation des courants dans les machines fictives (Figure 12, Figure 13) fait apparaître que l'ondulation est comparable dans la machine secondaire (valeur crête 60 A en Figure 10, 50 A en Figure 13) mais plus importante dans la machine principale. Par contre, l'observation des courants réels dans la machine met en évidence une réduction forte des ondulations, la machine homopolaire n'étant plus alimentée (Figure 14 à comparer avec la Figure 5). On a ainsi fortement amélioré la qualité des courants qui sont appelés par la machine réelle permettant ainsi de ne pas surdimensionner les interrupteurs.

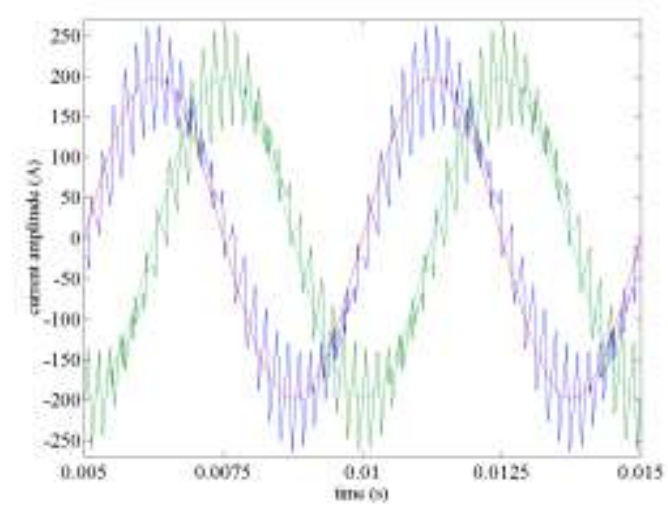


Figure 12. : Courants dans la machine principale

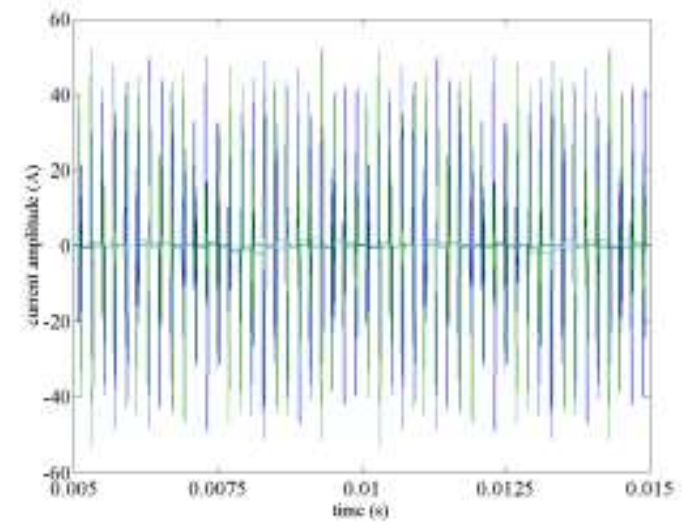

Figure 13. Courants dans la machine secondaire.

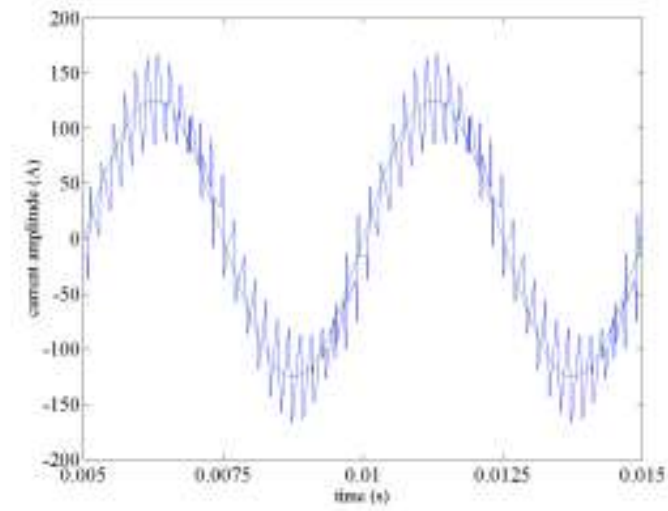

Figure 14. Courant de la phase réelle

\section{Application à un Cas Complexe}

\subsection{Structure étudiée}

Cette structure est composée de deux machines asynchrones pentaphasées connectées en série et alimentées par un onduleur 5 bras. Une connexion spécifique a été proposée pour permettre un fonctionnement indépendant des deux machines [GAT-00], [LEVI-03], [LEVI-04] : mise en série "croisée" (Figure 15). 
Machine $1 \quad$ Machine 2

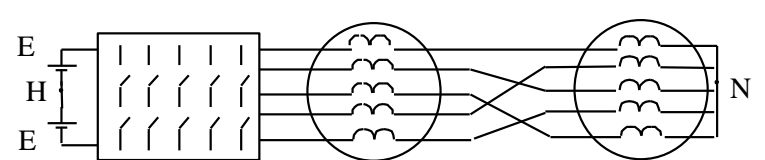

Figure 15. Onduleur 5 bras pour 2 machines asynchrones pentaphasées

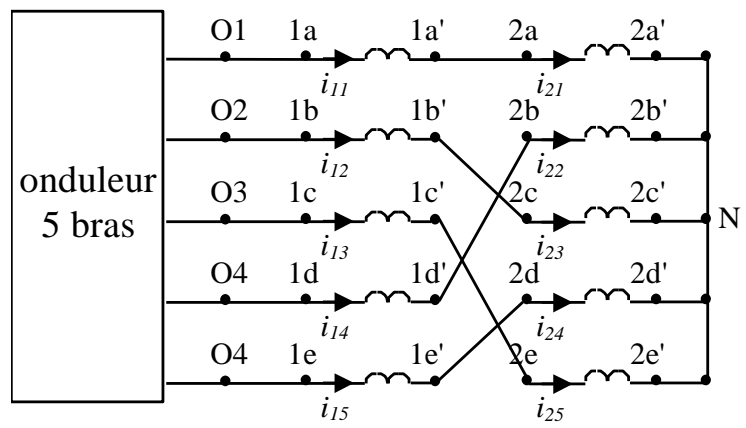

Figure 16. Structure de connexion

Cette connexion spécifique (Figure 16) peut se traduire par une relation matricielle entre les courants des deux machines :

$$
\left[\begin{array}{l}
i_{11} \\
i_{12} \\
i_{13} \\
i_{14} \\
i_{15}
\end{array}\right]=\left[\begin{array}{lllll}
1 & 0 & 0 & 0 & 0 \\
0 & 0 & 1 & 0 & 0 \\
0 & 0 & 0 & 0 & 1 \\
0 & 1 & 0 & 0 & 0 \\
0 & 0 & 0 & 1 & 0
\end{array}\right]\left[\begin{array}{l}
i_{21} \\
i_{22} \\
i_{23} \\
i_{24} \\
i_{25}
\end{array}\right]
$$

soit $\underline{i}_{m a 1_{-}} 12345=\left[L_{5}\right] \underline{i}_{m a 2_{-}} 12345$ avec $\left[L_{5}\right]$ matrice de liaison.

En effet cette connexion est caractérisée par la mise en série des enroulements $1 a 1 a^{\prime}$ et $2 a 2 a^{\prime}\left(i_{11}=i_{21}\right)$, des enroulements $1 b 2 b^{\prime}$ et $2 c 2 c^{\prime}\left(i_{12}=i_{23}\right)$ etc.

\subsection{Modélisation vectorielle}

Les deux machines pentaphasées vérifient les mêmes hypothèses que celles formulées pour la machine étudiée au paragraphe 3.2.. Par conséquent, aux deux machines sont associés les 3 mêmes sous-espaces propres, ces derniers dépendant simplement de la nature circulaire et symétrique de la matrice des inductances statoriques. On rappelle donc que l'on décompose l'espace de dimension 5 associé 
aux machines en 3 sous-espaces orthogonaux : deux plans $\mathrm{P}_{\mathrm{Y}}$ et $\mathrm{P}_{\mathrm{Z}}$ et une droite $\mathrm{D}_{\mathrm{W}}$. Tout vecteur peut alors s'écrire de façon unique :

$$
\vec{x}=\overrightarrow{x_{Y}}+\overrightarrow{x_{Z}}+\overrightarrow{x_{W}}
$$

avec $\overrightarrow{x_{Y}}, \overrightarrow{x_{Z}}$ et $\overrightarrow{x_{W}}$ vecteurs appartenant respectivement à $\mathrm{P}_{\mathrm{Y}}, \mathrm{P}_{Z}$ et $\mathrm{D}_{\mathrm{W}}$.

$$
\text { On définit } \underline{x}_{W Y Z}=\left[\begin{array}{l}
\overrightarrow{x_{W}} \\
\overrightarrow{x_{Y}}
\end{array}\right]=\left[\begin{array}{c}
x_{w} \\
x_{Y d} \\
x_{Y q} \\
x_{Z d} \\
x_{Z q}
\end{array}\right]
$$

La matrice de Concordia généralisée étendue à la dimension $5\left[\mathrm{C}_{5}\right]$ permet d'exprimer tout vecteur dans ce nouveau repère $(\mathrm{W}, \mathrm{Y}, \mathrm{Z})$. Dans ce cas, en tenant compte du fait que $\left[C_{5}\right]^{-1}=\left[C_{5}\right]^{t}$, on a par exemple pour les courants:

$$
\left[\begin{array}{l}
i_{1 W} \\
i_{1 Y d} \\
i_{1 Y q} \\
i_{1 Z d} \\
i_{1 Z q}
\end{array}\right]=\left[C_{5}\right]^{\mathrm{t}}\left[\begin{array}{l}
i_{11} \\
i_{12} \\
i_{13} \\
i_{14} \\
i_{15}
\end{array}\right] \text { et }\left[\begin{array}{c}
i_{2 W} \\
i_{2 Y d} \\
i_{2 Y q} \\
i_{2 Z d} \\
i_{2 Z q}
\end{array}\right]=\left[C_{5}\right]^{\mathrm{t}}\left[\begin{array}{c}
i_{21} \\
i_{22} \\
i_{23} \\
i_{24} \\
i_{25}
\end{array}\right]
$$

avec

$$
\left[C_{5}\right]=\sqrt{\frac{2}{5}}\left[\begin{array}{ccccc}
\frac{1}{\sqrt{2}} & 1 & 0 & 1 & 0 \\
\frac{1}{\sqrt{2}} & \cos \frac{2 \pi}{5} & \sin \frac{2 \pi}{5} & \cos \frac{4 \pi}{5} & \sin \frac{4 \pi}{5} \\
\frac{1}{\sqrt{2}} & \cos \frac{4 \pi}{5} & \sin \frac{4 \pi}{5} & \cos \frac{8 \pi}{5} & \sin \frac{8 \pi}{5} \\
\frac{1}{\sqrt{2}} & \cos \frac{6 \pi}{5} & \sin \frac{6 \pi}{5} & \cos \frac{12 \pi}{5} & \sin \frac{12 \pi}{5} \\
\frac{1}{\sqrt{2}} & \cos \frac{8 \pi}{5} & \sin \frac{8 \pi}{5} & \cos \frac{16 \pi}{5} & \sin \frac{16 \pi}{5}
\end{array}\right]
$$

soit

$$
\underline{i}_{m a 1 \_W Y Z}=\left[C_{5}\right]^{\mathrm{t}} \underline{i}_{m a 1 \_12345}
$$




$$
\underline{i}_{m a 2 \_W Y Z}=\left[C_{5}\right]^{\mathrm{t}} \underline{i}_{m a 2 \_12345}
$$

\subsection{Représentation SMM}

Cette transformation permet ainsi de retrouver pour chaque machine pentaphasée, ses machines fictives équivalentes MP (principale, associée au plan $\mathrm{P}_{\mathrm{Y}}$ ), MS (secondaire, associé au plan $\mathrm{P}_{\mathrm{Z}}$ ) et $\mathrm{MH}$ (homopolaire, associé à la droite $\mathrm{D}_{\mathrm{W}}$ ). Il est à noter que la connexion en étoile de la deuxième machine annule les composantes homopolaires des courants des deux machines.

A partir des relations des courants dans l'espace (W,Y,Z), et de celles exprimant la connexion entre les deux machines, on peut exprimer la connexion entre les machines fictives :

$$
\underline{i}_{m a 1_{-} W Y Z}=\left[C_{5}\right]^{-1}\left[L_{5}\right]\left[C_{5}\right] \underline{i}_{m a 2_{-} W Y Z}
$$

Après calculs, il vient :

$$
\left[C_{5}\right]^{-1}\left[L_{5}\right]\left[C_{5}\right]=\left[\begin{array}{ccccc}
1 & 0 & 0 & 0 & 0 \\
0 & 0 & 0 & 1 & 0 \\
0 & 0 & 0 & 0 & -1 \\
0 & 1 & 0 & 0 & 0 \\
0 & 0 & 1 & 0 & 0
\end{array}\right]
$$

Cela signifie que :

- $\quad i_{Y d}=i_{Z d}$ et $i_{Y q}=-i_{Z q}$ : la machine principale MP1 est en série avec la machine secondaire MS2 ;

- $\quad i_{Z d}=i_{Y d}$ et $i_{Z q}=i_{Y q}$ : la machine secondaire MS1 est en série avec la machine principale MP2.

Ces mises en série mènent donc à 4 variables d'état indépendantes, qui pourront être maîtrisées avec les 4 tensions entre phases issues de l'onduleur 5 bras. On peut ainsi gérer indépendamment les deux machines, par une gestion séparée de leur flux et de leur couples.

L'étude réalisée justifie alors a posteriori la connexion spécifique et originale trouvée par [GAT-00] en mettant en exergue les conditions de l'équivalence.

La représentation SMM s'explicite en différenciant les bobines équivalentes des conversions électromécaniques équivalentes (Figure 17). La mise en série d'enroulement apparaît alors comme un couplage électrique particulier. Cette notion 
a été clairement explicitée par la Représentation Energétique Macroscopique (REM) pour la mise en série d'enroulements d'induit de machines à courant continu dans le cadre d'un système de traction [Bou-02].

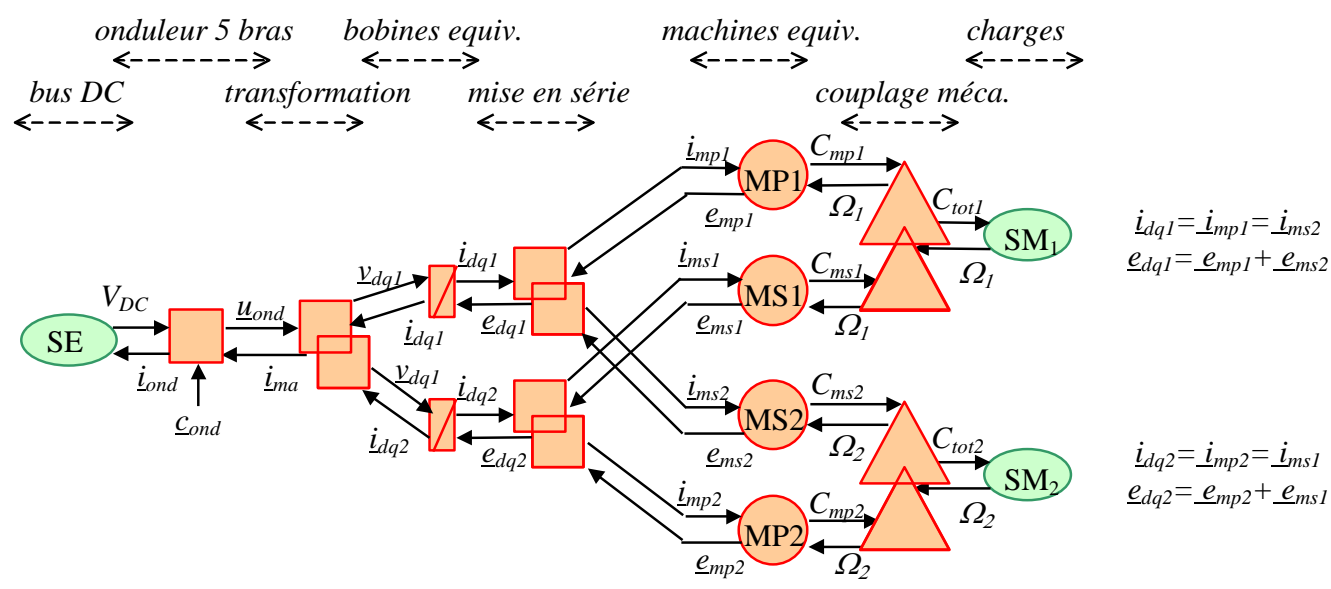

Figure 17. Représentation SMM du système étudié

\section{Conclusion}

La combinaison des outils du formalisme SMM et du vecteur d'espace généralisé mène à la décomposition d'une machine polyphasée en machines fictives équivalentes. Cette représentation permet alors une analyse intéressante de ce type de machine. Elle peut conduire à des structures de commande originales et performantes. Ainsi dans le premier cas étudié, l'origine principale des ondulations de courant ayant été déterminée (courants dans la machine homopolaire), une commande utilisant uniquement des vecteurs tension de l'onduleur sans composante homoplaire a été définie. Dans le deuxième cas, l'obtention d'un système découplé plus simple permettra d'implanter des structures de commande déjà élaborées pour le contrôle de machines à courant continu avec induits en série [Bou-02]. Bien entendu, cette simplicité n'existe que dans les espaces liés aux machines fictives. Elle disparaît si l'on décrit la commande dans l'espace naturel associé aux machines.

Plus généralement, la mise en forme avec le formalisme graphique SMM permet de faire apparaître plus facilement l'équivalence d'un système avec d'autres plus connus. Il s'en suit l'application de lois de commande déjà éprouvées.

Cette approche peut bien sûr être étendue à d'autres machines [SEM-03]. Parmi les travaux déjà engagés dans le projet SMM, on peut citer les travaux sur les machines double étoile [HAD-01] et les machines double alimentation [VID-03]. 


\section{Références}

[Bou-02] A. Bouscayrol, Ph. Delarue, E. Semail, J. P. Hautier, J. N. Verhille, "Application de la macro-modélisation à la représentation énergétique d'un système de traction multimachine", Revue Internationale de Génie Electrique, vol. 5 n³-4, octobre 2002 pp 431-453 (article commun L2EP Lille, Matra Transport International).

[CoA-01] C. Coates, D. Platt, V. Gosbell, "Performance Evaluation of a Nine-Phase Synchronous Reluctance Drives”, Proc. of IEEE-IAS'01, Chicago, September 2001, CDROM.

[GAT-00] S. GATARIC, "A polyphase cartesian Vector Approach To control of Polyphase AC Machines”, Proc. of IEEE-IAS'00, Rome, October 2000, CD-ROM.

[HAD-01] D. HADIOUCHE, "Contribution à l'étude de la machine asynchrone double étoile : modélisation, alimentation et structure", thèse de doctorat de l'UHP, Nancy 1, décembre 2001.

[JAH-80] T. JAHNS, "Improved reliability in solid state ac drives by means of multiple independent phase-drive units", IEEE Trans. on Industry Applications, vol. 16, May-June 1980, pp 321-331.

[Kes-02] X. Kestelyn, E. Semail, J. P. Hautier, "Vectorial Multi-machine modelling for a five phase machine", Proc. of ICEM'02, Bruges, August 2002, CD-ROM.

[Kes -03] X. Kestelyn, "Modélisation Vectorielle Multimachines pour la commande des ensembles Convertisseurs-Machines polyphasés", Thèse de doctorat de l'USTL, Lille, 2003.

[Levi-03], E. Levi, M. Jones, S.N. Vukosavic, H.A. Toliyat, "A Five-Phase Two-Machine Vector Controlled Induction Motor Drive Supplied from a Single Inverter", Proc. of EPE'03, Toulouse, September 2003, CD-ROM.

[Levi-04], E. Levi, M. Jones, S.N. Vukosavic, H.A. Toliyat, "A Novel Concept of a Multiphase, Multimotor Vector Controlled Drive System Supplied From a Single Voltage Source Inverter", IEEE Trans. On Power Electronics, Viol. 19, no. 2, March 2004.

[LYR-01] R. LYRA., T. LiPO, "Torque Density Improvement in a Six-Phase Induction Motor With Third Harmonic Current Injection”, Proc. of IEEE-IAS'01, Chicago, September 2001.

[Mar-02] J. P. Martin, E. Semail, S. Pierfederici, A. Bouscayrol., F. Meibody-Tabar, B. DAVAT, "Space Vector Control of 5-phase PMSM supplied by q H-bridge VSIs", Proc. of ElectrIMACS'02, Montreal, August 2002, CD-ROM

[Mou-98] N. Moubayed, F. Meibody-Tabar, B. Davat, "Alimentation par deux onduleurs de tension d'une machine synchrone double étoile", Revue Internationale de Génie Electrique, vol. 1, $\mathrm{n}^{\circ}$ 4, 1998, pp. 457-470.

[Rob-02] E. Robert-Dehault, M. F. Benkhoris, E. Semail, "Study of a 5-phase synchronous machine fed by PWM inverters under fault conditions", ICEM'02, Bruges, August 2002, CD-ROM

[SEM-00] E. Semail, "Outils et méthodologie d'étude des systèmes électriques polyphasés. 
Généralisation de la méthode des vecteurs d'espace", Thèse de doctorat de l'USTL, Lille, 2000.

[SEm-01] E. Semail, X. Kestelyn, "Modélisation multimachine d'une machine synchrone polyphasée ", EF'2001, Nancy, 2001, pp. 203-208.

[Sem-03] E. Semail, A. Bouscayrol, J. P. Hautier, "Vectorial formalism for analysis and design of polyphase synchronous machines", EPJ Applied Physics, vol. 22, no. 3, June 2003, pp. 207-220.

[SMM-00A] (projet SMM du GdR SDSE) A. Bouscayrol, B. Davat, B. De Fornel, B. François, J. P. Hautier, F. Meibody-Tabar, M. Pietrzak-David, "Multimachine Multiconverter System: application for electromechanical drives", European Physics Journal - Applied Physics, vol. 10, no. 2, May 2000, pp-131-147.

[SMM-00B] (PROJET SMM du GdR SDSE) A. Bouscayrol, B. Davat, B. DE Fornel, B. François, J. P. Hautier, F. Meibody-Tabar, M. Pietrzak-David, "Multimachine Multiconverter Systems for drives: analysis of coupling by a global modeling", Proc. of IEEE-IAS anual meeting 2000, Rome, October 2000, CD-ROM

[SMM 03] (PROJet SMM du GdR ME²MS) A. Bouscayrol, B. Davat, B. De Fornel, B. François, J. P. Hautier, F. Meibody-Tabar, E. Monmasson, M. Pietrzak-David, H. RAZIK, M. F. BENKHORIS, "Control structure for multimachine multiconverter systems with upstream coupling", Mathematics and Computers in Simulation, vol. 63, no3-5, November 2003, pp. 261-270

[Tol-00] H. A. Toliyat, S. Ruhe, X. Huansheng, "A DSP-Based vector Control of FivePhase Synchronous Reluctance Motor”, IEEE-IAS'00, Rome, October 2000, CD-ROM.

[Vid-03] P. E. VidAl, M. PieTRZAK-DAVID, B. DE Fornel, "Stator flux oriented control of a doubly fed induction machine", Proc. of EPE'2003, Toulouse, September 2003, CDROM. 